\title{
Image Fusion Using Tensor Decomposition and Coefficient Combining Scheme
}

\author{
Mugdha S. Rane \\ Dept. of Electronics and Telecommunication Engg. \\ JSPM's Rajarshi Shahu College of Engineering \\ University of Pune, India
}

\begin{abstract}
In this paper, an effective image fusion method is proposed for creating a highly informative fused image through merging multiple images. The proposed method is based on higher order singular value decomposition (HOSVD). Since image fusion depends on local information of source images, the proposed algorithm simply groups together similar patches of source images to constitute the fused image by processing the divided 3D stack rather than the whole tensor. Then it computes the sum of absolute values of the coefficients (SAVC) from HOSVD of sub-tensors for activity-level measurement to evaluate the quality of the related image patch; and a novel sigmoid-function-like coefficient-combining scheme is applied to construct the fused result. Experimental results show that the proposed algorithm is an alternative fusion approach for multi-modal and multi-focus images.
\end{abstract}

Index Terms-Coefficient-combining strategy, singular value decomposition (SVD), image fusion, sigmoid function.

\section{INTRODUCTION}

Image fusion is a process of combining relevant information from two or more images of the same scene to get the more informative image [1]. Input images could be multi sensor, multimodal, multi focal or multi-temporal. Image fusion is an important technique for various image processing and computer vision applications such as feature extraction and target recognition. The fused image can provide more comprehensive information about the scene which is more useful for human and machine perception [3]-[6]. Image fusion find application in the area of navigation guidance, object detection and recognition, medical diagnosis, satellite imaging for remote sensing, rob vision, military and civilian surveillance, etc. For instance, the performance of feature extraction algorithms can be improved by fusing multi-spectral remote sensing images. The fusion of multi-exposure images can be used for digital photography. In these applications, a good image fusion method has the following properties. First, it can preserve most of the useful information of different images. Second, it does not produce artifacts. Third, it is robust to imperfect conditions such as mis-registration and noise.

A large number of image fusion methods [3]-[9] have been proposed in literature. Some are in spatial domain and some are in transform domain. Among these methods, multi-scale image fusion [5], [6] and data-driven image fusion [7], [8] are very successful methods. They focus on different data representations and different image fusion

\author{
Prof. Dr. D. S. Bormane \\ Dept. of Electronics and Telecommunication Engg. \\ JSPM's Rajarshi Shahu College of Engineering \\ University of Pune, India
}

rules to guide the fusion of coefficients. The major advantage of these methods is that they can well preserve the details of different source images. However, these kinds of methods may produce spatial distortions.

The fusion process [1] consists of three basic stages: Image Acquisition, Image Registration and Image Fusion. Image Acquisition is the process of acquiring images from one or several image sensors. Image registration is the process of establishing a point-by-point correspondence between multiple images depicting the same scene or different scene. Before the image fusion algorithm is applied to the source images, image registration is used to ensure the correspondence between the pixels in the input images. Lastly, the image fusion process is used to combine the relevant information from the set of source images, into a single image.

The advantages of HOSVD, high-dimensional data representation and feature extraction, motivate to propose the image fusion algorithm based on transform domain using Higher order SVD [8]. HOSVD is an extension of the SVD to higher order dimensions. It is not optimal tensor decomposition in the sense of least squares data fitting and has not the truncation property of the SVD, where truncating the first singular values [9] permits to find the best -rank approximation of a given matrix. Despite this, the approximation obtained is not far from the optimal one and can be computed much faster [9], [10]. In fact, the computation of HOSVD [2] does not require iterative alternating least squares algorithms, but needs standard SVD computation only.

It is worthwhile to highlight several aspects of the proposed transform domain-based approach here.

1) Source images refer to the same scene (multi-focus images or multi-modal images); this paper constructs them into a tensor and employs the HOSVD technique to extract their features simultaneously [8]. Furthermore, an algorithm picks out informative image patches of source images to constitute the fused image by processing the divided subtensors rather than the whole tensor. 


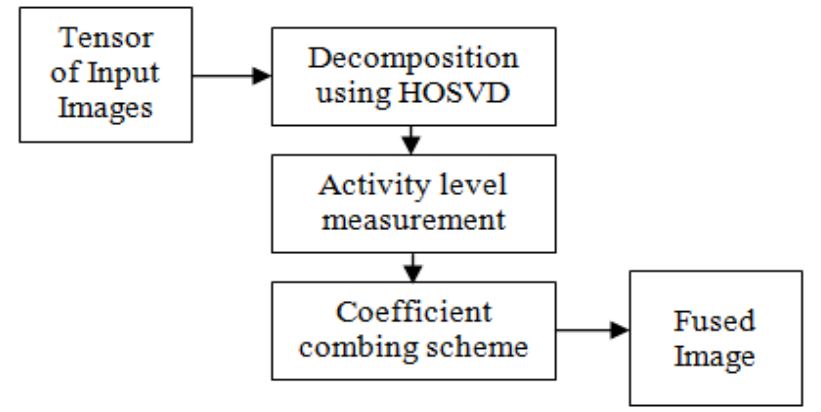

Fig. 1. Block schematic of proposed image fusion method.

2) A slice of the core tensor yielded from HOSVD of subtensors reflects the quality of the related image patch. Unlike the conventional activity-level measurements, which apply the absolute value of a single coefficient to evaluate the corresponding pixel, this paper employs the sum of absolute values of coefficients (SAVC) [8] as the activity-level measurement of the related patch.

3) To adapt to different activity-level measurements, this paper proposes a flexible sigmoid-function-like coefficientcombining scheme [8], which incorporates the choose-max scheme and the weighted average scheme.

A novel image fusion method extends the proposed algorithm to fuse color images refer to multi-modal (PET and MR) images. The article is structured as follows. In Section II, we present the topics related to work as Tensor decomposition using HOSVD, sigmoid function like coefficient-combining scheme. In Section III, we describe the algorithm based on HOSVD and show how it is used for gray-scale as well as colour images. Section IV describes the results in terms of performance measures also shows the effect of patch size on performance. Finally, Section V concludes the article.

\section{RELATED WORK}

\section{A. Tensor Decomposition}

Tensor decomposition was studied in psychometric data analysis during the 1960 s, when data sets having more than two dimensions (generally called "three-way data sets") became widely used [10]. In this section, we present the definitions and properties of HOSVD [2], highlighting its difference with respect to standard SVD [10].

Tensors are generalizations of vectors (that have one index) and matrices (that have two indices) to an arbitrary number of indices. A tensor can be represented as a multidimensional array of numerical values [2]. Here, several notations and operations of tensors [8], [10] will be used in the rest of this paper. We denote tensors as calligraphic letters (A, B , etc.), matrices as capital letters (A, $\mathrm{B}$ etc.), and vectors as small letters ( $\mathrm{a}, \mathrm{b}$ etc.).
1) An Nth order tensor is an object with $\mathrm{N}$ indices, i.e. $A \in \mathrm{R}^{\mathrm{I}_{1} \times \mathrm{I}_{2} \times \ldots \times \mathrm{I}_{\mathrm{N}}}$.

2) An nth-mode vector of an $\left(\mathrm{I}_{1} \times \mathrm{I}_{2} \times \ldots \times \mathrm{I}_{\mathrm{N}}\right)$ dimensional tensor $A$ is an n-dimensional vector obtained by varying index in but fixing the other indices.

3) The nth-mode product of a tensor $A \in R^{I_{1} \times I_{2} \times \ldots \times I_{N}}$ and a matrix $\mathrm{U} \in R^{I_{n} \times I_{n}}$ along the nth mode is denoted by,

$$
B=A \times{ }_{n} U \in R^{I_{1} \times I_{2} \times \ldots \times I_{N}}
$$

with elements,

$$
\begin{aligned}
b_{i_{1}, i_{2}, \ldots, i_{n-1}, j_{n}, i_{n+1}, \ldots, i_{N}} & \\
= & \sum_{i_{n}=1}^{I_{n}} a_{i_{1}, i_{2}, \ldots, i_{n-1}, i_{n}, i_{n+1}, \ldots, i_{N}} \cdot u_{j_{n}, i_{n}}
\end{aligned}
$$

where $u_{j_{n}, i_{n}}$ stands for the $\left(j_{n}, i_{n}\right)^{\text {th }}$ element of unitary matrix $\mathrm{U}$, and $a_{i \_1, i \_2, ., i \_(n-1), i \_n, i \_(n+1), \ldots, i \_N}$ represents the $\left(i_{1}, i_{2}, \ldots, i_{n-1}, i_{n}, i_{n+1}, \ldots, i_{N}\right)$ element of tensor $\mathrm{A}$.

4) The nth-mode matricization of a tensor $A$ is an operation where the nth-mode vectors of $\mathrm{A}$ are aligned as the columns of a matrix, which is denoted by $A(n)$.

5) HOSVD of a tensor $A \in \mathrm{R}^{\mathrm{I}_{1} \times \mathrm{I}_{2} \times \ldots \times \mathrm{I}_{\mathrm{N}}}$ is given by,

$$
\begin{aligned}
& \qquad A=\sum_{\text {where } \sum \in \mathrm{R}^{\mathrm{I}_{1} \times \mathrm{I}_{2} \times \ldots \times \mathrm{I}_{\mathrm{N}}} \text { is the core tensor that satisfies }}^{\mathrm{x}_{1}} \mathrm{U}_{1} \mathrm{x}_{2} \mathrm{U}_{2} \ldots \mathrm{x}_{\mathrm{N}} \mathrm{U}_{\mathrm{N}} \\
& \text { is the con }
\end{aligned}
$$
the all orthogonality conditions.

The Singular Value Decomposition (SVD) of a matrix gives us important information about a matrix such as its rank, an orthonormal basis for the column or row space, and reduction to diagonal form. In applications, especially those involving multiway data analysis, information about the rank and reduction of tensors to have fewer nonzero entries are useful concepts to try to extend to higher dimensions.

Some applications need orthogonality of the matrices for better interpretation of the data.

HOSVD is data-driven decomposition technique and can extract the features of multiple slices of the decomposed tensor simultaneously. Therefore, two source images are constructed into a tensor $A$ with $(M \times N \times 2)$ dimensions (i.e., with three modes: the row, the column, and the label of the source image order), and HOSVD is employed to extract the related features (i.e., to obtain the decomposition coefficients). Although HOSVD is used to obtain the decomposition coefficients (or extract features) of multiple images there are two important differences. On the one hand, since image fusion relies on local information of source images, we form $(\widetilde{M} \times \widetilde{N} \times 2)$ dimensional subtensor $B_{i}(1)$ and $B_{i}(2)$ separately from the two source images and perform the HOSVD of $A_{i}$. So that informative image patches are picked out to piece together the final fused image. On the other hand, unlike the conventional method, which directly employs the slice of the core tensor as the features of the corresponding image, we use the nth-mode product of the core tensor and the third-mode factor matrix 
to reflect the quality of the related image patch for the purpose of constructing the final fused result.

\section{B. Quality Measures}

The application area of image fusion determines the evaluation method of fusion. In image fusion application, the aim of fusion is to process the significant parts of source images, for instance, the edges and regions with high contrast. This type of evaluation is based on the perceptual information. On the other hand, some quantitative measures can be used for performance evaluation of fusion method. In the quantitative performance evaluation [8], [11- 13], we evaluate fusion on the basis of statistical parameters of fused image. Several parameters can be used for evaluating the performance of fusion algorithm. In the proposed work we have used following performance evaluation metrics namely fusion factor, fusion symmetry, information entropy, mutual information, standard deviation, overall cross entropy, PSNR, image quality index, $\mathrm{Q}_{\mathrm{AB} / \mathrm{F}}$ metric etc. These performance metrics are briefly introduced as follows:

\section{1) Entropy:}

It reflects the amount of information in the fused image. The larger the EN is, the more information the image carries. Entropy is an index to evaluate the information quality of an image. If the entropy value becomes higher after fusion, it is an indication that the information quality has increased and the fusion performance has improved.

$$
\text { Entropy }=-\sum_{i=0}^{G} p(i) \log _{2}\{p(i)\}
$$

where $G$ is the number of gray levels in the image's histogram (255 for a typical 8-bit image), and p(i) is the normalized frequency of occurrence of each gray level, i.e., the histogram of the image. To sum up the self-information of each gray level from the image, the average information content (entropy) is estimated in the units of bits per pixel. It should be noted that entropy is also sensitive to noise and other unwanted rapid fluctuations.

\section{2) Mutual Information:}

The mutual information $\mathrm{I}(\mathrm{A}, \mathrm{B})$ is used to measure the similarity of image intensity distribution between images A and B. Image histograms can be used to obtain distribution probabilities. Higher the value of I (A,B) better the similarity between $\mathrm{A}$ and $\mathrm{B}$, and thus a better fusion algorithm. Mutual Information is defined as,

$$
I(A, B)=\sum_{a, b} P_{A B}(a, b) \times \frac{\log \left(P_{A B}(a, b)\right)}{\left(P_{A}(a) P_{B}(b)\right)}
$$

where, $\mathrm{P}_{\mathrm{AB}}(\mathrm{a}, \mathrm{b})$ is the joint distribution probability, $\mathrm{P}_{\mathrm{A}}$ (a) and $\mathrm{P}_{\mathrm{B}}(\mathrm{b})$ are the distribution probabilities of $\mathrm{A}$ and $\mathrm{B}$, respectively.

\section{3) Overall cross entropy:}

It can reflect the difference between the two source images and the fused image. It is determined by entropy and mutual information. Therefore, the smaller the OCE gives the better fusion result that is obtained.

$$
O C E=\frac{\left(H_{1}+H_{2}\right)}{2}
$$

where, $H_{1}$ is Entropy $(F)-\mathrm{I}(A, F)$ and $\mathrm{H}_{2}$ is $\operatorname{Entropy}(F)-\mathrm{I}$ $(B, F)$. Here, $A, B$ are the source images and $F$ is the fused image.

\section{4) Fusion factor:}

It is the sum of the mutual information of source images and fused image.

$$
F F=I(A, F)+I(B, F)
$$

where $I(A, F)$ and $I(B, F)$ are mutual information between source images and fused image. Mutual information is a basic concept of information theory measuring the amount of information that one image contains about another. Thus, higher value of fusion factor gives more information about image.

\section{5) Peak SNR:}

The fused image is looked upon as the ideal image (signal) plus the noise image (difference between the ideal image and the fused image). The larger the SNR value, the better the fused result.

$$
P S N R=10 \log _{10}\left(\frac{P_{e a k^{2}}}{M S E}\right)
$$

Here Peak is the maximum possible value is having every bit as 1 , i.e $11111111=255$. And $M S E$ is nothing but mean squared error.

\section{6) Image quality metrics:}

Natural image signals would be highly structured and their pixels reveal strong dependencies. These dependencies would carry vital information about the structure of the object. It compares local patterns of pixel intensities that have been normalized for luminance and contrast.

$$
\operatorname{IQM}(A, B)=\frac{\left(2 \mu_{A} \mu_{B}+C_{1}\right)\left(2 \sigma_{A B}+C_{2}\right)}{\left(\mu_{A}^{2}+\mu_{B}^{2}+C_{1}\right)\left(\sigma_{A}^{2}+\sigma_{B}^{2}+C_{2}\right)}
$$

where $\mu_{A}, \mu_{B}$ are the mean intensities of reference image and fused image respectively. $\sigma$ is the standard deviation and $\sigma_{\mathrm{AB}}$ is the covariance between these two images. The larger the IQM value, better similarities in the fused result.

\section{7) $Q_{A B / F}$ metric:}

It considers the amount of edge information transferred from the input images to the fused image [14]. It should be close to 1 as much as possible. Having $\mathrm{Q}_{\mathrm{AF}}$ and $\mathrm{Q}_{\mathrm{BF}}$ for $(\mathrm{M} \times \mathrm{N})$ size images, a normalised weighted performance metric 
$\mathrm{Q}_{\mathrm{AB} / \mathrm{F}}$ of a given fusion process that operates on images $\mathrm{A}$ and $\mathrm{B}$, and produces $\mathrm{F}$ is obtained as follows:

$Q_{A B / F}=$

$$
\frac{\sum_{m=1}^{M} \sum_{n=1}^{N} Q_{A F}(m, n) w_{A}(m, n)+Q_{B F}(m, n) w_{B}(m, n)}{\sum_{i=1}^{M} \sum_{j=1}^{N}\left(w_{A}(i, j)+w_{B}(i, j)\right)}
$$

Note that the edge preservation values, $\mathrm{Q}_{\mathrm{AF}}$ and $\mathrm{Q}_{\mathrm{BF}}$ are weighted by $\mathrm{w}_{A}(\mathrm{~m}, \mathrm{n})$ and $\mathrm{w}_{\mathrm{B}}(\mathrm{m}, \mathrm{n})$, respectively. In general, edge preservation values which correspond to pixels with high edge strength should influence $\mathrm{Q}_{\mathrm{AB} / \mathrm{F}}$ more than those of relatively low edge strength.

\section{IMAGE FUSION ALGORITHM}

A new image fusion algorithm is developed according to the steps below. To facilitate the description, we begin with two $(\mathrm{M} \times \mathrm{N})$ dimensional gray (multi-focus) images then the extension of the proposed algorithm is for color (multimodal) image fusion.

Steps for multi-focus image fusion:

1. Construct the tensor of two input images of same scene with $(256 \times 256 \times 2)$ dimensions.

2. Form a sub-tensor of patches and apply Higher Order SVD transform simultaneously.

3. Find Sum of Absolute Value of the Coefficients for activity level measurement.

4. Apply fusion rule i.e. coefficient combining scheme.

5. Evaluate the performance of fused image in terms of quality measures.

Steps for multi-modal image fusion:

1. Construct the tensor of two input images taken from different modal with $(256 \times 256 \times 4)$ dimensions; out of these two inputs, one is PET (colour) image $(256 \times 256 \times 3)$ and another is MR (gray-scale) image $(256 \times 256)$ of human brain.

2. Form a sub-tensor of patches and apply Higher Order SVD transform simultaneously.

3. Find Sum of Absolute Value of the Coefficients for activity level measurement.

4. Apply fusion rule i.e. coefficient combining scheme.

5. Evaluate the performance of fused image in terms of quality measures.

\section{A. Initialization}

In this, we are going to take two input images. For multi-focus image fusion, the first one 'far focused image' and second is 'near focused image' and for multi-modal image fusion, the first on 'PET image' and second is 'MR image' of human brain. We are going to concatenate the two images and construct in to form of tensor $(M \times N \times 2)$ and $(M \times N \times 4)$ for multi-focus and multi-modal images respectively by using tensor tool box with is special tool box available for tensor based application. To construct the fused result conveniently, we employ the sub-tensor $(\widetilde{\mathrm{M}} \times \widetilde{\mathrm{N}} \times$ 2 ) of two gray-scale image patches and $(\widetilde{M} \times \widetilde{N} \times 4)$ of PET and MR image patches. Here we choose patch size is equal to 4 i.e. $(2 \times 2)$. Image fusion depends on local information of source images rather than total information, this paper picks out informative image patches of source images to constitute the fused image by processing the divided sub-tensors rather than the whole tensor. Then it employs the HOSVD technique to extract their features simultaneously.

\section{B. Decomposition of Coefficients}

HOSVD is one of most efficient data-driven decomposition techniques [8]-[10] and can extract the features of multiple slices of the decomposed tensor simultaneously. For $\mathrm{i}=1,2, \ldots, \mathrm{I}$, let the HOSVD of divided sub-tensor $A_{i}$ be given by,

$$
S=A_{i} \times{ }_{1} U^{(1)^{T}} \times_{2} U^{(2)^{T}} \times_{3} U^{(3)^{T}}
$$

here core tensor $S$ is all-orthogonal and ordered. All orthogonality [2] means that the "horizontal slices" of $S$ (fixing i constant) are mutually orthogonal with respect to the inner product. The ordering condition is simply a convention that fixes a particular ordering of the columns of $U^{(n)}$, much like in the case of matrix SVD.

The sub-tensors $A_{i}$ using two image patches $\mathrm{B}_{\mathrm{i}}(1)$ and $\mathrm{B}_{\mathrm{i}}(2)$ separately from the two source images and perform the HOSVD of $A_{i}$, so that informative image patches are picked out to piece together the final fused image. On the other hand, unlike the conventional method, which directly employs the slice of the core tensor as the features of the corresponding image, we use the nth mode product of the core tensor and the third-mode factor matrix to reflect the quality of the related image patch for the purpose of constructing the final fused result from the product above more conveniently.

\section{Activity-level Measurement}

It is commonly thought that the magnitude (absolute value) of the decomposed coefficient is consistent with the related local energy, which implies that the larger the absolute value of the coefficient is, the more information the corresponding pixel contains. Therefore, many transform domain fusion methods employ the absolute value of the coefficient as the activity-level measurement of the corresponding pixel. Borrowing the idea but unlike it, this paper defines the SAVC as the activity-level measurement of the related image patch to evaluate its quality.

Based on coefficient matrix $A_{i}(:,:, l)$, the activity-level measurement of image patch $B_{i}(l)$ is defined as, 


$$
e_{i}(l)=\sum_{m=1}^{\widetilde{M}} \sum_{n=1}^{\widetilde{N}} A_{i}(:,:, l), \quad l=1,2
$$

According to these activity-level measurements, coefficient matrices $A_{i}(:,:, 1)$ and $A_{i}(:,:, 2)$ are merged to obtain a new coefficient matrix $D_{i}$, i.e.

$D_{i}=$

$$
\begin{aligned}
& \frac{1}{1+e^{\left(-k \ln \left(\frac{e_{i}(1)}{e_{i}(2)}\right)\right)} \times \sum_{i}(:,:,: 1)+} \\
& \frac{e^{\left(-k \ln \left(\frac{e_{i}(1)}{e_{i}(2)}\right)\right)}}{1+e^{\left(-k \ln \left(\frac{e_{i}(1)}{e_{i}(2)}\right)\right)}} \times \sum_{i}(:,:,: 2)
\end{aligned}
$$

where $k$ is the shrink factor of sigmoid function.

Fused image patch is

$$
F_{i}=U_{i} \times D_{i} \times V_{i}^{T}=B_{i}, \quad i=1,2, \ldots I
$$

\section{Sigmoid function}

To derive the proposed coefficient-combining scheme, we first consider all possibilities [8] from fig 2: 1) In the same sub-tensor, the image patch with an even higher SAVC value contains more rich information or is of higher quality; thus, it should be directly selected (choose-max strategy) as the final fused result of the corresponding sub-tensor. 2) If the SAVCs of both image patches are close to each other, then they have approximate image quality, and thus, their weighted average should be used as the ultimate fused result of the sub-tensor.

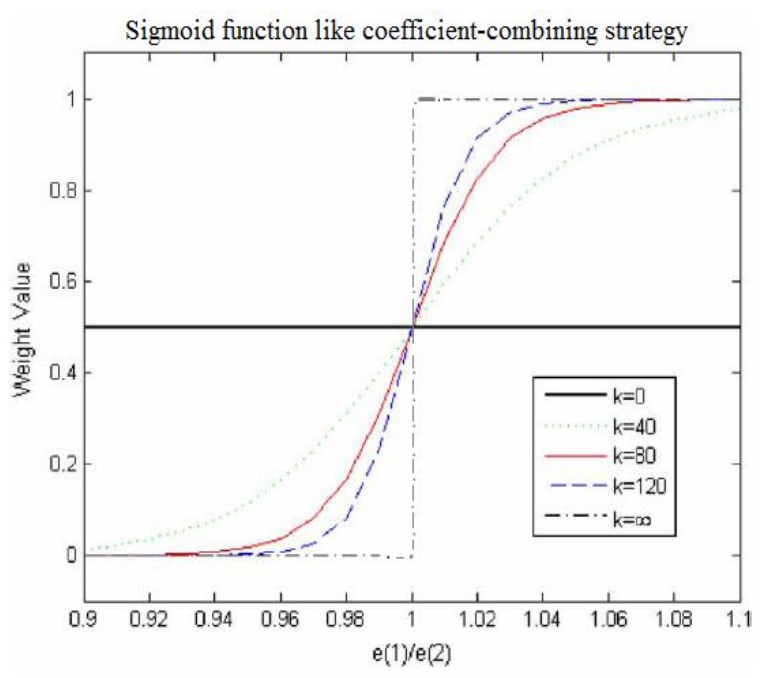

Fig. 2. Sigmoid Function with different shrink factors

However, for the first case, when two adjacent image patches are chosen, respectively, from different source images, it will cause discontinuous gap pixels between adjacent patches of the fused image. Therefore, a choosemax strategy with the smoothing function should be designed. In order to attain the aim above, this paper designs a novel sigmoid-function-like coefficient-combining scheme to adapt to different cases. From fig. 2, When $k=\infty$, the first situation is mapped into the flat region of the sigmoid function's range, and then, the approximate choose-max scheme works. When $k=80$, the second one is projected into its steep region and in this case, the weighted average scheme works.

Fused image patch:

$$
\begin{aligned}
& F_{i}=B_{i}(1) \\
& F_{i}=\left[B_{i}(1)+B_{i}(2)\right] / 2 \\
& F_{i}=B_{i}(2)
\end{aligned}
$$$$
\begin{aligned}
& \ldots \text { If } e_{i}(1)>e_{i}(2) \\
& \ldots \text { If } e_{i}(1)=e_{i}(2) \\
& \ldots \text { If } e_{i}(2)>e_{i}(1)
\end{aligned}
$$

\section{EXPERIMENTAL RESULTS}

To verify the proposed method, it is tested on computer vision, medical images and compared with the conventional DWT, DCT, PCA, and LAP-based approaches [3]-[6], [8].

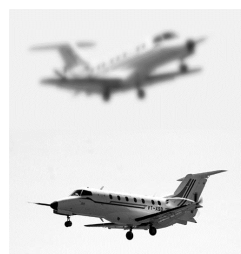

(a)

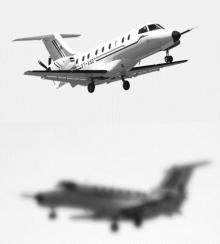

(b)

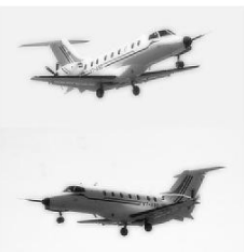

(c)
Fig 3. Saras_Plane (a) Input image1 (b) Input image2 (c) Fused image

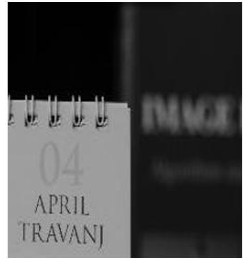

(a)

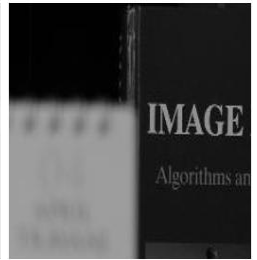

(b)

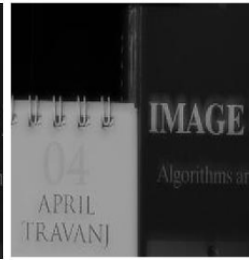

(c)
Fig 4. Cal_Img (a) Input image1 (b) Input image2 (c) Fused image

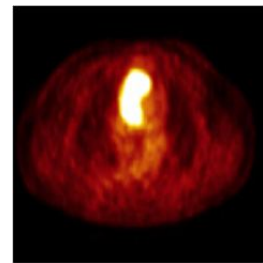

(a)

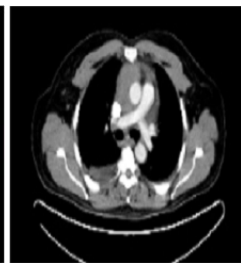

(b)

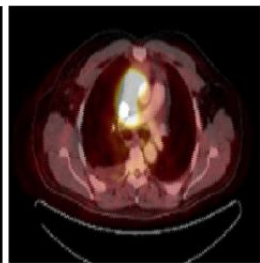

(c)
Fig 5. Medical Image (a) PET image1 (b) MR image2 (c) Fused image 
A. Performance Evaluation of the Proposed Fusion Algorithm

Note that, except for $\mathrm{Q}^{\mathrm{AB} / \mathrm{F}}$, EN, and OCE, other metrics require the ideal or original image as the reference image.

Performance results for multi-focus image fusion:

\begin{tabular}{|c|c|c|}
\hline \multirow{2}{*}{$\begin{array}{l}\text { Performance } \\
\text { Parameters }\end{array}$} & \multicolumn{2}{|c|}{ Results of proposed scheme } \\
\hline & $\begin{array}{c}k=120 \\
\text { for fig. } 3\end{array}$ & $\begin{array}{c}k=80 \\
\text { for fig. } 3\end{array}$ \\
\hline Entropy & 10.9250 & 10.5250 \\
\hline Mutual Information & 2.7669 & 2.7669 \\
\hline Fusion Factor & 10.2533 & 9.8533 \\
\hline Standard Deviation & 44.0207 & 43.6207 \\
\hline Mean Square Error & 0.0450 & 0.0675 \\
\hline Peak SNR & 77.1216 & 75.3606 \\
\hline $\mathrm{Q}_{\mathrm{AB} / \mathrm{F}}$ & 1.7045 & 1.3045 \\
\hline Image Quality Index & 16.6204 & 1700204 \\
\hline Overall Cross Entropy & 8.2971 & 7.8971 \\
\hline
\end{tabular}

Performance results for multi-modal image fusion:

\begin{tabular}{|c|c|c|}
\hline \multirow{2}{*}{$\begin{array}{l}\text { Performance } \\
\text { Parameters }\end{array}$} & \multicolumn{2}{|c|}{ Results of proposed scheme } \\
\hline & $\begin{array}{c}k=120 \\
\text { for fig. } 5\end{array}$ & $\begin{array}{c}k=80 \\
\text { for fig. } 5\end{array}$ \\
\hline Entropy & 13.9535 & 9.3023 \\
\hline Mutual Information & 2.2295 & 2.2295 \\
\hline Fusion Factor & 10.6874 & 7.1249 \\
\hline Standard Deviation & 52.6620 & 35.1080 \\
\hline Mean Square Error & 0.2858 & 0.4287 \\
\hline Peak SNR & 57.1808 & 55.4199 \\
\hline $\mathrm{Q}_{\mathrm{AB} / \mathrm{F}}$ & 1.2443 & 0.8443 \\
\hline Image Quality Index & 1.0907 & 0.7271 \\
\hline Overall Cross Entropy & 11.7019 & 7.8013 \\
\hline
\end{tabular}

\section{B. Effect of Patch Size on the Proposed Algorithm}

In this experiment, the effect of patch size [8] on the performance of the proposed algorithm is investigated when $k=120$ (take "fig.3" for example). When the patch size varies from 4 to 12 , the related $\mathrm{EN}, \mathrm{Q}_{\mathrm{AB} / \mathrm{F}}$ and $\mathrm{OCE}$ performance metrics are given in Fig. 6(a)-(c), respectively.

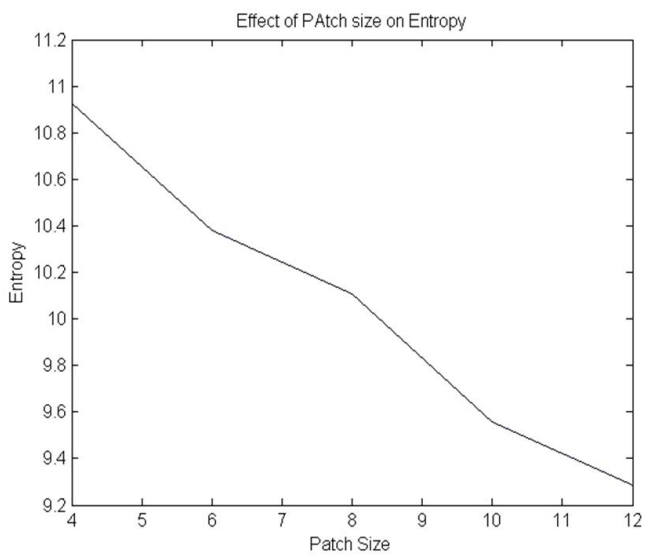

Fig. 6a. Effect of Patch size on Entropy

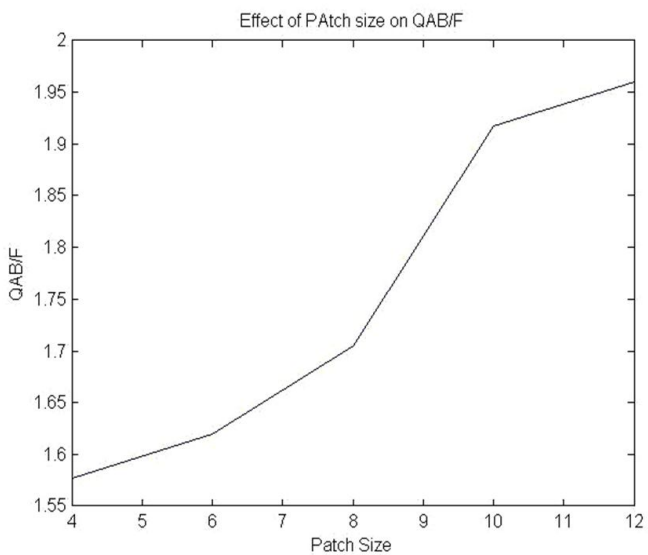

Fig. 6b. Effect of Patch size on $\mathrm{Q}^{\mathrm{AB} / \mathrm{F}}$

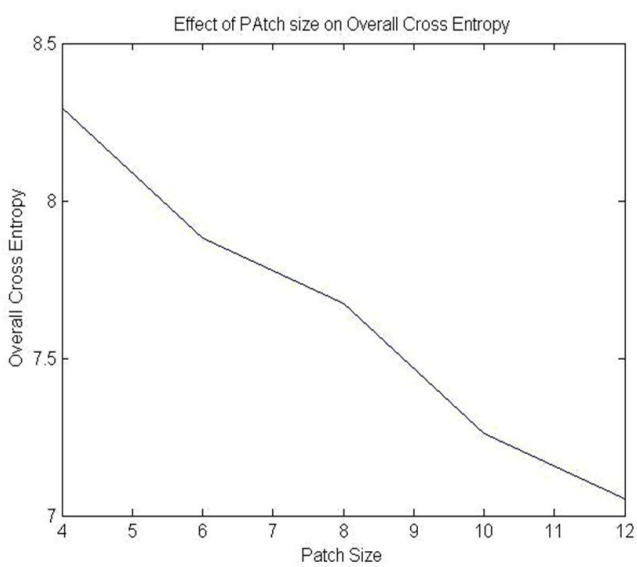

Fig. 6c. Effect of Patch size on Overall Cross Entropy

1) The $Q_{A B / F}$ value increases as the patch size increases; 2) As the patch size increases, the EN value drops and 3) The OCE value decreases with the increase in patch size. 


\section{CONCLUSION}

The experiments show that the proposed algorithm is an alternative image fusion method for both multi-focus and multi-modal images. The success of the proposed algorithm lies in the following: 1) HOSVD, a fully data-driven technique, is an efficient tool for high-dimensional data decomposition and feature extraction; 2) the SAVC is a feasible activity-level measurement for evaluating the quality of image patches; and 3) the sigmoid-function-based coefficient-combining strategy incorporates the conventional choose-max strategy and the weighted average strategy and thus adapts to different activity levels.

An effective and fast image fusion algorithm using tensor decomposition and coefficient-combining scheme has been proposed. Finally, experimental results show that the proposed transform domain algorithm is having some spatial distortions but it is tolerable for multi-modal medical image fusion. Also It is an alternative color image fusion approach.

\section{REFERENCES}

[1] A. A. Goshtasby and S. Nikolov, "Image fusion: Advances in the state of the art," Inf. Fusion, vol. 8, no. 2, pp. 114-118, Apr. 2007.

[2] G. Bergqvist and E. G. Larsson, "The higher-order singular value decomposition: Theory and application," IEEE Signal Process. Mag., vol. 27, no. 3, pp. 151-154, May 2010.

[3] E. H. Adelson, C. H. Anderson, J. R. Bergen, P. J. Burt, and J. Qgden, "Pyramid methods in signal processing," RCA Eng., vol. 29, no. 6, pp. 33-41, Nov./Dec. 1984

[4] H. Yesou, Y. Besnus, and J. Rolet, "Extraction of spectral information from Landsat TM data and merger with SPOT panchromatic imagery- A contribution to the study of geological structures," ISPRS J. Photogramm. Remote Sens., vol. 48, no. 5, pp 23-36, Oct. 1993.

[5] H. Li, S. Manjunath, and S. Mitra, "Multi sensor image fusion using the wavelet transform," Graph. Models Image Process., vol. 57, no. 3, pp. 235-245, May 1995.

[6] J. Tang, "A contrast based image fusion technique in the DCT domain," Digit. Signal Process., vol. 14, no. 3, pp. 218-226, May 2004.

[7] D. Looney and D. Mandic, "Multiscale image fusion using complex extensions of EMD,” IEEE Trans. Signal Process., vol. 57, no. 4, pp 1626-1630, Apr. 2009

[8] J. Liang, Y. He, D. Liu, and X. Zeng, "Image fusion using higher order singular value decomposition," IEEE Trans. Image Process., vol. 21, no. 5, May 2012.

[9] L. De Lathauwer, B. De Moor, and J. Vandewalle, "A multilinear singular value decomposition,” SIAM J. Matrix Anal. Appl., vol. 21, no. 4, pp. 1253-1278, Mar.-May 2000.

[10] R. Costantini, L. Sbaiz, and S. Susstrunk, "Higher order SVD analysis for dynamic texture synthesis," IEEE Trans. Image Process., vol. 17, no. 1, pp. 42-52, Jan. 2008.

[11]C. S. Xydeas and V. Petrovic, "Objective image fusion performance measure,” Electron. Lett., vol. 36, no. 4, pp. 308-309, Feb. 2000.

[12] Mohammed Hossny, Saeid Nahavandi, Douglas Creighton, Asim Bhatti and Marwa Hassan, "Image Fusion Metrics: Evolution in a Nutshell", pp. 443-450, IEEE 2013.

[13]Z.Wang and A. Bovik, "A universal image quality index," IEEE Signal Process. Lett., vol. 9, no. 3, pp. 81-84, Mar. 2002.
[14] M. Kumar and S. Dass, "A total variation-based algorithm for pixel level image fusion,” IEEE Trans. Image Process., vol. 18, no. 9, Sep. 2009.

[15] Hongbo Wu, YanqiuXing, "Pixel-based Image Fusion Using Wavelet Transform for SPOT and ETM+ Image", IEEE International Conference on Image Processing, pp. 936-940, IEEE 2010.

[16] Hugo R. Albuquerque, Tsang Ing Ren and George D. C. Cavalcanti, "Image Fusion Combining Frequency Domain Techniques Based on Focus", 24th International Conference on Tools with Artificial Intelligence, pp. 757-762, IEEE 2012.

[17] Shutao Li, Member, IEEE, Xudong Kang, Student Member, IEEE, and Jianwen $\mathrm{Hu}$, "Image Fusion with Guided Filtering", IEEE Trans. on Image Processing Vol. 22, No. 7, July 2013.

[18] M. Haardt, F. Roemer, and G. Del Galdo, "Higher-order SVD-based subspace estimation to improve the parameter estimation accuracy in multidimensional harmonic retrieval problems," IEEE Trans. Signal Process., vol. 56, no. 7, pt. 2, pp. 3198-3213, Jul. 2008.

[19] Ajit Rajwade, Student Member, IEEE, Anand Rangarajan, Member, IEEE, and Arunava Banerjee, Member, IEEE, "Image Denoising Using the HOSVD", IEEE Trans. on Pattern Analysis and Machine Intelligence Vol. 35, No. 4, April 2013.

[20] R. Shen, I. Cheng, J. Shi, and A. Basu, "Generalized random walks for fusion of multi-exposure images," IEEE Trans. Image Process., vol. 20, no. 12, Dec. 2011.

[21] Brandon Miles, Ismail Ben Ayed, Member, IEEE, Max W. K. Law, Greg Garvin, Aaron Fenster, Senior Member, IEEE, and Shuo Li, "Spine Image Fusion Via Graph Cuts", IEEE Trans. on Biomedical Engg, Vol. 60, No. 7, July 2013. 